\title{
LE DISPOSITIF ICONOTEXTUEL DES MÈMES : CLICHÉS VARIABLES ET SUBVERSION DES GENRES
}

\section{ICONOTEXTUAL MEMETIC DEVICES: UNSTABLE CLICHES AND SUBVERSION OF GENRES}

\author{
Laura Goudet \\ Normandie Univ, UNIROUEN, ERIAC (EA 4705), Rouen, França
}

\begin{abstract}
Résumé: Les mèmes d'Advice Animals représentent des animaux et des humains qui incarnent des clichés comportementaux, exprimés par des discours renforcés par les titres (mèmonymes) et la décontextualisation de l'image originale. Bien que ces mèmes contiennent des énoncés inscrits dans des genres délimités (conseils, microrécits personnels, parodies...), ils les remettent en question : le conseil est malveillant, la parodie ne fait que reprendre les paroles de l'autre. Les archétypes ne suivent pas non plus les règles établies, ni pour les mèmes animaliers ni pour les stéréotypes humains : le cliché animal se renouvelle par contradiction, et l'évocation des stéréotypes sociaux s'accompagne de leur décomposition.
\end{abstract}

Mots clés: Analyse de discours numérique ; Cliché ; Mémétique ; Sémiotique.

Abstract: Advice Animal memes resort to the use of animals and humans embodying behavioral clichés, and whose discourse is reinforced by their titles ('memonymes') as well as the decontextualization of the initial image. Although these memes feature discourse belonging to specific genres (advice, personal stories, satire...), they challenge them: advice is ludicrous, and satire only recuperate others' discourse and replace it in another context. Archetypes break the existing rules, whether animal or human stereotypes: animal clichés update through selfcontradiction, and social preconceptions are simultaneously summoned and dismantled.

Keywords: Clichés; Digital discourse analysis; Memetics; Semiotics.

\section{Introduction}

Les mèmes internet sont envisagés (à tort) comme une prolongation de la théorie de la propagation comportementale chez les animaux par l'imitation de pairs. Ce que R. Dawkins définissait comme une "unité d'information contenue dans un cerveau, échangeable au sein d'une société» (DAWKINS, 1976, p. 192) a évolué vers l'acception "même internet». Ce sont des dispositifs souvent composés d'une image assortis d'une légende, dont un bon exemple est les lolcats. Ces illustrations représentent un chat, qui s'accompagne d'un commentaire prétendument émis par lui. Ce principe autoréplicatif, à fort ancrage culturel, a évolué grâce aux outils créés par et pour les consommateurs de ce phénomène. 
Certains méméticiens dénoncent les mèmes internet, et les décrivent ainsi :

des objets sans nom, mais des objets qui démontraient la capacité de certains contenus à se répliquer de façon naturellement massive, sans aucune intervention humaine globale, mais uniquement en reposant sur des microcomportements élémentaires consistants à copier-coller, à tripatouiller les images, à télécharger, à partager, bref à faire des choses très simples, quasiment dépourvues d'intention ou de créativité (JOUXTEL, 2013).

Par abus de langage, les mèmes internet ont adopté le même nom que les "éléments de code culturel reconnaissable et reproductible» ${ }^{1}$. Je vais m'attacher à montrer ici qu'ils sont non seulement de microcomportements, mais aussi des faisceaux de codes culturels en- et hors-ligne, qui évoluent, se disséminent et servent à communiquer en connivence avec ceux qui partagent cette sous-culture, et bouleversent ainsi les codes stéréotypiques établis.

Le terme de dispositif est emprunté à $M$. Foucault pour décrire les mèmes. La définition d'un dispositif est «tout ce qui $a$, d'une manière ou d'une autre, la capacité de capturer, d'orienter, de déterminer, d'intercepter, de modeler, de contrôler et d'assurer les gestes, les conduites, les opinions et les discours des êtres vivants» (AGAMBEN, 2014, p. 13). Les créations iconotextuelles où image et texte confluent assoient un archétype comportemental, conceptuel, associé à un genre discursif (conseil ou parodie) par les multiples itérations de la même illustration, appelée image-macro. Les centaines, voire les milliers d'exemples de chaque mème convergent vers la corroboration du stéréotype original.

La condensation sémiotique et discursive provoque l'archétypie, qui contribue à l'intelligibilité rapide de ces discours formatés. Seule la légende change, mais pas le cadre thématique de ces images. Le dispositif comprend la police employée, la scission du texte de part et d'autre de l'illustration, et le type de discours utilisé. Le respect des conventions tacites de création des mèmes iconotextuels montre la littératie numérique des créateurs. Je propose la notion de "cliché variable" pour parler de ces mèmes, qui se renouvellent en renversant leur valeur, ou en contestant les archétypes sociaux.

La source primaire principale est le site de curation Know Your Meme (KYM). C'est une encyclopédie de mèmes internet, très documentée. Les internautes y confirment l'existence d'un mème, après une étape de

${ }^{1}$ Définition de P. Jouxtel, sur le site de la Société Francophone de Mémétique, disponible à l'adresse : <http://www.memetique.org/2013/09/les-memes-dans-le-larousse-2014/>. Dernier accès le : 21 juin 2016. 
soumission à la base de données. Chaque entrée contient un historique et est mise à jour régulièrement. Reddit et 4Chan, deux communautés virtuelles extrêmement fréquentées ${ }^{2}$ seront aussi mentionnées. 4Chan est un site plus confidentiel, mais il a été le berceau de nombre de mèmes internet, comme le rickrolling ou même "Pedobear». Ce site très controversé à cause de sa population composée de trolls est le pendant obscur de Reddit.

La première partie est consacrée aux éléments qui constituent l'identité d'un mème. D'abord, j'établirai une typologie à partir de leur titre, qui est le plus souvent en dehors du dispositif iconotextuel, puis je décrirai le processus de baptême d'un mème, ainsi que la décontextualisation caractéristique de certains de ces Advice Animals.

Je passerai ensuite à l'analyse du type de discours présent dans ce corpus. La notion de conseil est inhérente aux Advice Animals, et la première section s'attachera à montrer la façon dont le genre injonctif évolue dans ce contexte. J'examinerai la parodie et le microrécit, dans les mèmes de critique du quotidien, puis dans les anecdotes personnelles.

Dans la troisième partie, les stéréotypes seront examinés : les animaux et l'évolution des archétypes animaliers ont une grande part dans leur renouvellement. J'aborderai l'évocation des idées reçues hors-ligne, à travers les mèmes qui renversent les stéréotypes sociétaux, comme "Successful Black Man».

\section{Genèse mémétique}

Dans cette partie, je m'attache à la description formelle des mèmes d'Advice Animals, en établissant d'abord une typologie des noms de mèmes, ou mèmonymes. J'évoquerai l'aspect collaboratif d'un tel baptême, qui rassemble les communautés internet autour d'un stéréotype marquant. Enfin, le phénomène de décontextualisation de l'image, qui la vide de son sens premier afin qu'elle incarne un archétype, sera examiné.

\subsection{Typologie mèmonymique}

Bien que les Advice Animals soient des documents iconographiques, ils ont tous un nom composé de deux à trois mots, qui permet de les désigner ou de les retrouver facilement sur les sites de curation (KYM, Meme Generator...). Ces taxons ont des caractéristiques communes : ils

\footnotetext{
${ }^{2}$ Respectivement 234 et 27 millions de visiteurs uniques par mois.
} 
sont souvent plus longs que des anthroponymes (de deux à quatre mots), et constituent de réelles étiquettes descriptives. Ils dépeignent le plus souvent l'image et l'archétype correspondant.

Le modèle de désignation le plus fréquent est la création d'un nom composé [nom + hyperonyme]. Il se décline sous diverses formes : concept incarné par un animal ("Courage Wolf», "Depression Dog»), ou groupe nominal qui expose soit la situation d'énonciation ("Confession Bear»), soit le contexte de l'image («First Day on the Internet Kid»).

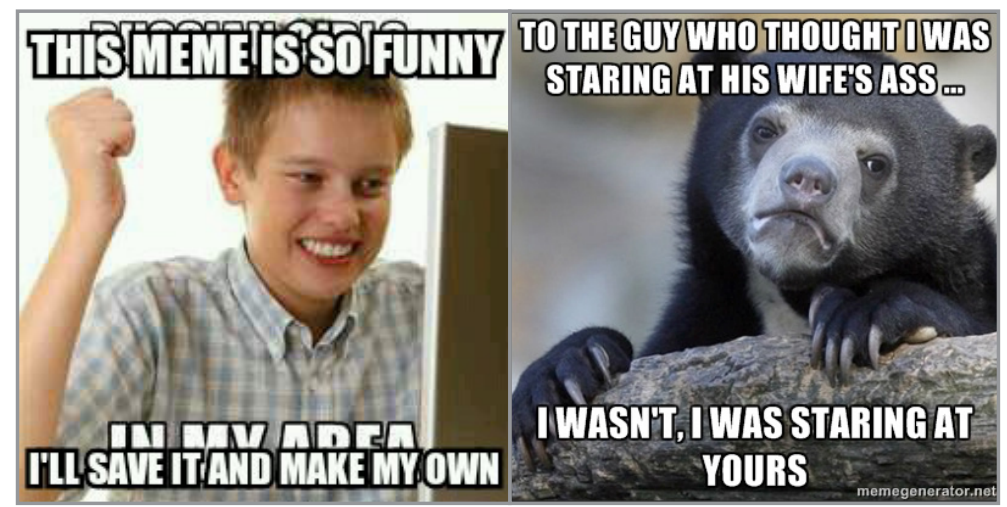

Illustration 1: Exemples de "First Day on the Internet Kid " et "Confession Bear " . Source : Imgur.

La syntaxe de la langue anglaise place le cliché ou thème général avant le second terme, bien plus générique, dans la construction du syntagme mèmonymique. La seconde partie peut être un hyperonyme animalier, comme "Dog", ou une classification d'occupation ou de genre, pour les humains («Professor» ou «Girl»), qui devient individuatif une fois qu’il rejoint le mèmonyme. L'immense majorité des Advice Animals utilise ce type de dénomination, puisqu'ils sont les plus descriptifs ( $82 \%$ des titres sur KYM).

Le patron [caractéristique + prénom] est minoritaire $(8 \%$ des mèmes sur KYM). Ce modèle mèmonymique est réservé aux Advice Animals qui comportent des archétypes humains, comme «Bad Luck Brian» ou «Sudden Clarity Clarence ». Le choix du prénom, et non d'un hyperonyme, provoque une proximité plus grande avec le personnage de l'illustration. Les mèmes de cette catégorie utilisent souvent la dialectique de l'aveu personnel, pour «Minor Mistake Marvin», où les internautes racontent les petits incidents 
de la vie qu'ils ont provoqués. Ils peuvent également créer une entité à part entière, avec un discours codifié, à l'instar de "Bad Luck Brian", associé à l'humour scatologique ("Bends over to pick up girls' [sic] dropped book/ shits", 'se penche pour ramasser un livre qu'une fille a fait tomberldéfeque's). L'archétype de ces personnages représente toutes les manifestations de cette caractéristique. Cela les éloigne des mèmes où l'hyperonyme les restreint à une seule situation, comme l'enseignement pour "Rasta Science Teacher» ou «Engineering Professor", surtout dans le cas des mèmes humains. Le prénom les rapproche curieusement des mèmes où les hyperonymes sont des noms d'animaux : "Minor Mistake Marvin» et "Confession Bear" contiennent tous deux des récits personnels (traités dans la seconde partie du présent article); Marvin comme l'ours représentent donc l'énonciateurcréateur du mème, puisque l'archétype incarne le thème général de son discours.

Des citations utilisées dans la légende peuvent également apparaitre dans les titres, comme «Bold Move Cotton» ('Stratégie audacieuse, Cotton'), "I too like to live dangerously" ('Moi aussi, j'aime vivre dangereusement'). Ces mèmonymes n'apparaissent que pour les mèmes constitués d'une image d'un film relativement récent, et d'une citation (cf. Illustration 2). La seconde partie de la légende est un extrait d'Austin Powers, et coïncide avec le moment d'où l'image est tirée.

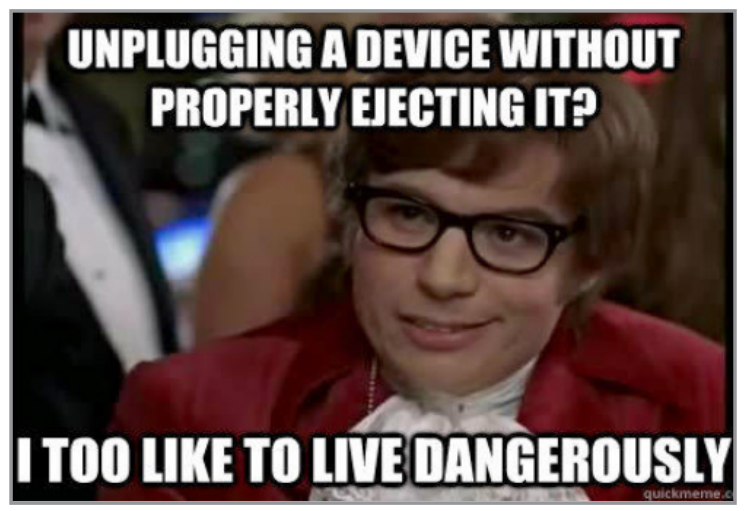

Illustration 2 : Exemple du mème "I too like to live dangerously " . Source : KYM.

\footnotetext{
${ }^{3}$ Image disponible à l'adresse $:<$ http://knowyourmeme.com/photos/271202-bad-luck-brian> Dernier accès le : 20 juin 2016.
} 
Ces mèmonymes (et les mèmes qu'ils représentent) se différencient des autres à cause de leur faible indexicalité vers le mème lui-même : ils renvoient à la source extérieure, et à la mémoire de la scène originale, connue des amateurs de ce film. Le décalage humoristique provient de l'utilisation de la chute "I too like to live dangerously", après une amorce plutôt triviale (ici, «retirer un périphérique [à un ordinateur] sans l'éjecter»). Ces mèmes reproduisent la scène originale, où le personnage d'Austin Powers joue très mal au blackjack avec l'antagoniste du film, et justifie son action par la phrase qui donnera son titre au mème. Les Advice Animals de ce type représentent $8 \%$ des entrées sur KYM.

La dernière catégorie enfin, avec seulement $2 \%$ des Advice Animals de KYM, est l'attaque ad personam où une célébrité est prise à partie à cause d'une faille utilisée comme symbole d'erreurs similaires (voir Illustration 3). Ainsi, "Wrong Lyrics Christina» fut créé quand la chanteuse Christina Aguilera s'est trompée dans les paroles de l'hymne national américain lors du Superbowl 2011. "Liberal Douche Garofalo», qui satirise les opinions politiques de gauche, a émergé quand l'humoriste et activiste J. Garofalo a déchainé les critiques en affirmant que les membres du Tea Party américain étaient tous racistes en 2009. L'animateur de l'émission "Man vs Wild ", Bear Grylls est tourné en dérision pour avoir consommé son urine lors de ses aventures télévisuelles, et n'apparaît que dans des mèmes à l'humour scatologique, avec la même conclusion, "Better drink my own piss» (“ [je ferais mieux de] boire mon urine']). Cette citation est l'autre nom de ce mème, ce qui le place entre la catégorie du mèmonyme citationnel, et celui de l'attaque ad personam.

\footnotetext{
${ }^{4}$ Aussi connue sous le titre "Born Survivor», "Ultimate Survival», "Survival Game» et "Seul face à la nature» en France, cette émission de Discovery Channel 4 fut produite de 2006 à 2011.
} 


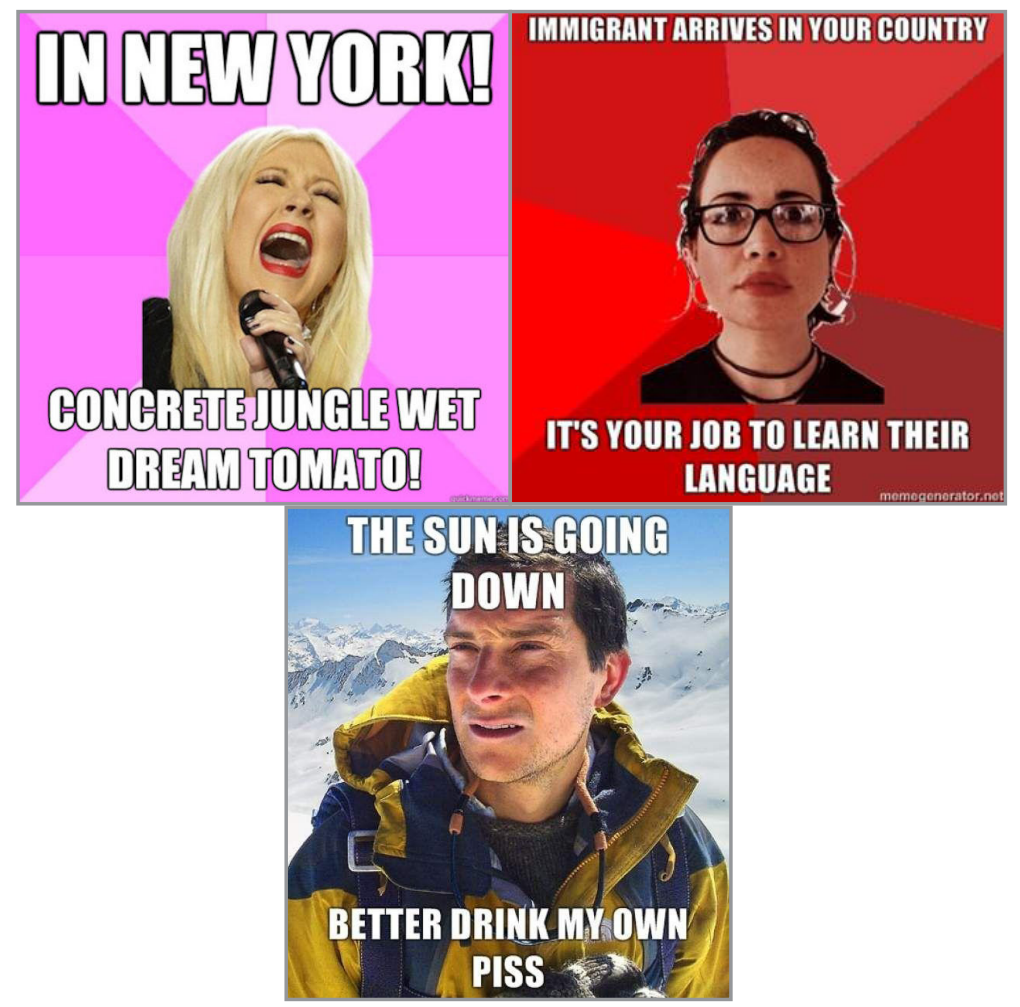

Illustration 3 : Exemples de «Wrong Lyrics Christina», «Liberal Douche Garofalo» et «Bear Grylls" .

Source : KYM.

Ces mèmes contiennent un discours parodique sur l'attitude de ces célébrités critiquées par les internautes, ce qui les enferme dans un cadre comportemental constitué grâce à la mémoire d'un prédiscours remarqué par les créateurs.

Les mèmonymes confirment le principe de Quine, "pas d'entité sans identité", et son son corollaire chez Geach, "pas d'identité sans entité» (QUINE apud GEACH, 1973, p. 23). Les titres représentent autant de rubriques thématiques qui indiquent au lecteur néophyte ce qu'il trouvera dans les manifestations de chaque mème, ou qui permettent de les retrouver facilement, sur les sites d'archivage ou de création. Ces mèmonymes appartiennent entièrement au dispositif qu'ils accompagnent, et constituent des guides d'écriture pour les occurrences futures. 
La question du choix mèmonymique est cruciale pour la genèse d'un Advice Animal, et elle implique souvent une négociation pour trouver le titre le plus descriptif.

\subsection{Choix des de titres}

Le créateur du dispositif prime n'est pas le seul acteur des communautés virtuelles à décider de l'adoption d'un mèmonyme. L'exemple «Ordinary Muslim Man» montre que l'auteur original, un membre de Reddit, a donné un nom efficace au personnage : "Introducing Ordinary Muslim Man" (' [je vous] présente l'Homme musulman lambda)'. Cette image initiale (Illustration 4) a été postée sur Imgur et son lien, partagé immédiatement sur Reddit dans la section $r / p i c s$ (photographies), et non dans r/AdviceAnimals, puisque cette illustration ne faisait pas encore partie du fonds culturel mémétique.

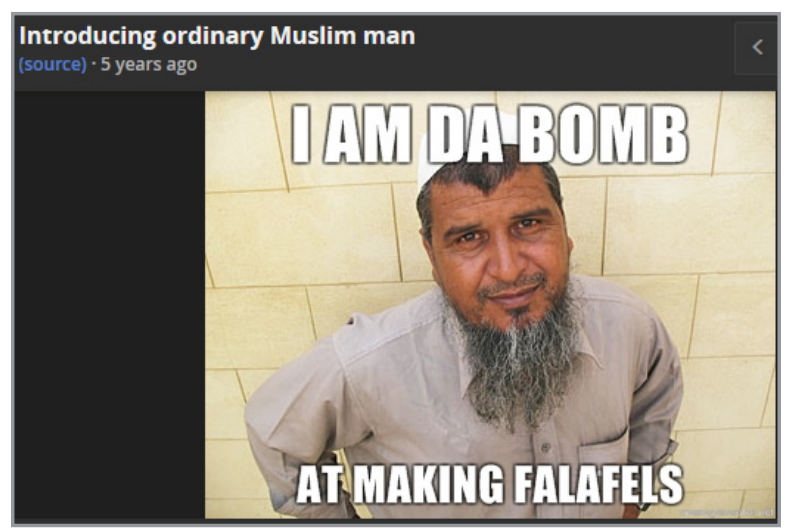

Illustration 4 : première occurrence du mème "Ordinary Muslim Man» . Source : Imgur apud Reddit.

Une fois trouvés, ces noms ne changent pas, surtout quand les sites de curation les emploient. Des contre-exemples existent, surtout lorsqu'une communauté virtuelle crée un mème qui est ensuite popularisé auprès d'un plus grand public. Ainsi, le nom initial de «Scumbag Steve» ('Steve Salaud ${ }^{6}$ )

\footnotetext{
5 Fil de discussion disponible à l'adresse : <https://www.reddit.com/r/pics/comments/ew7zf/ introducing_ordinary_muslim_man/>. Dernier accès le : 18 juin 2016.

${ }^{6}$ Le souci de conservation euphonique autant que de sémantisme anime les choix traductionnels
} 
était «Petty Theft Paul» ('Paul Rapine') sur 4Chan avant que son mèmonyme actuel $^{7}$ ne perce, y compris sur KYM. L'Illustration 5 montre qu'à peine un mois après que le mème a été popularisé sur Reddit, les internautes se querellaient sur son appellation, dans les commentaires d'un blog où figurait un des exemples du mème.

\section{Teutorix \\ FEBRUARY 20, 2011 AT 3:48 AM}

Petty theft Paul is the best meme ever, I heard the real guy is mad as hell.

Punk1119

FEBRUARY 20, 2011 AT 1:50 PM

It's Scumbag Steve, get it right.

\section{Anonymouse}

FEBRUARY 20, 2011 AT 8:49 PM

i think it was petty theft paul before it became scumbag steve. does it really matter?

Illustration 5 : Commentaires divergents sur le mèmonyme «Scumbag Steve» . Source : The Troll Cave.

Les cas les plus intéressants, linguistiquement parlant, sont ceux où les fils de discussion qui proposent un nouveau personnage à l'approbation de la communauté servent également à lui trouver un nom. Pour rester sur l'exemple de "Scumbag Steve», sa première apparition ${ }^{8}$ sur Reddit où il était présenté dans une série d'images date de 2011. Comme il n'avait pas de taxon, ce fil de discussion a donné lieu à une plateforme d'expression où les internautes ont proposé diverses désignations (Illustration 6).

de l'auteur. Les traductions de citations de films proviennent des versions françaises.

${ }^{7}$ Il n'y a que 161 résultats sur Google pour «Petty Theft Paul», contre 536000 pour «Scumbag Steve" au 22 juin 2016. Ce dernier nom est aussi celui de l'entrée de ce mème sur KYM.

${ }^{8}$ Disponible à l’adresse : <https://www.reddit.com/r/funny/comments/f65ra/i_hated_this_ dude/?sort=best $>$. Dernier accès le : 14 juin 2016 . 


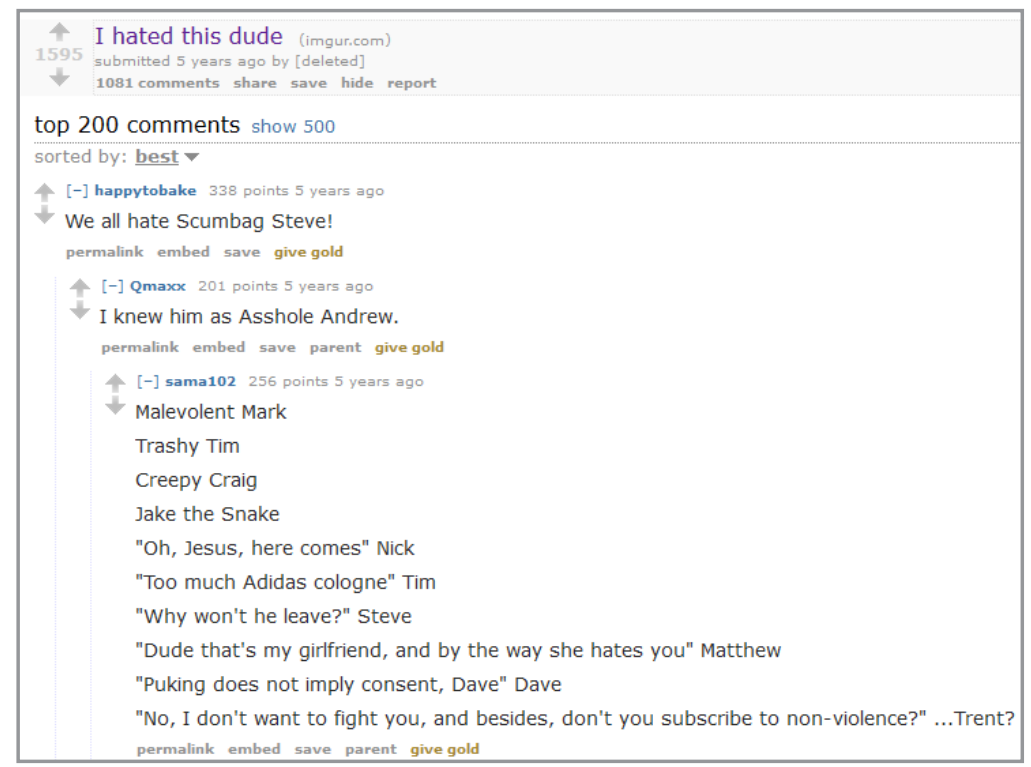

Illustration 6 : Premiers commentaires sur le fil de discussion pour«Scumbag Steve» . Source : Reddit.

Ces propositions sont principalement des jeux euphoniques sur des allitérations entre le prénom et la caractéristique. On exprime souvent cette dernière grâce à un adjectif, comme dans «Shit-shack Shane» ('Shane fait chier') ou "Jackass John." ('John Neu-neu'), mais aussi par des phrases complètes, comme « «Too much Adidas cologne» Tim» ou ««Why won’t he leave?» Steve». Ces exemples sont des réactions à la présence d'une personne qui correspondrait au stéréotype exprimé par «Scumbag Steve», mais, moins percutants, ils sont disqualifiés. En revanche, le choix sur lequel Reddit s'est arrêté, "Scumbag Steve», est proposé dans le message le mieux noté par la communauté. Le mot «scumbag» est moins fréquent que d'autres candidats, comme «jackass" (respectivement 211 contre 534 occurrences dans le COCA (Davies, 2008)). Il n'est ni trop rare, comme «shit-shack» (hapax du corpus COCA), ni préalablement accolé à un élément culturel, comme "Jackass", titre d'une émission de télé-réalité à succès aux ÉtatsUnis. Sa relative rareté, couplée au fait qu'on puisse lui associer un prénom plutôt courant pour créer l'euphonie, en a fait un candidat tout trouvé pour désigner cet archétype. 
Ces baptêmes ancrent ces mèmes dans la culture internet : avoir un nom, c'est avoir une identité. C'est également avoir une place dans l'environnement immédiat des internautes, comme en atteste un autre message sur ce fil de discussion : "I have never seen a meme I could relate too more. I knew SO many kids like this in High School" "Je n'ai jamais vu un mème que je pouvais autant comprendre. Je connaissais TELLEMENT de gens comme ça au lycée'). Les clichés des mèmes occupent une place hybride, entre existence en- et hors ligne, et leur décontextualisation fait partie intégrante de leur succès.

\subsection{Décontextualisation mémétique}

Les Advice Animals sont des dérivés d'un mème initial où l'image d'un chien était présentée sur un fond constitué d'une roue de couleur. Cet arrière-plan est commun à bon nombre de mèmes de cette catégorie comme en atteste le collage utilisé sur KYM pour symboliser l'entrée dictionnairique (Illustration 7). Les animaux sauvages présentés (loup, pingouin, et même vélociraptor) n'attirent pas les internautes en tant que tels. Ils incarnent autant de symboles d'un comportement (être riche ou gauche en société...) ou d'un sentiment (le courage, la folie...). La décontextualisation des images est primordiale pour pouvoir les libérer de leur existence exomémétique.

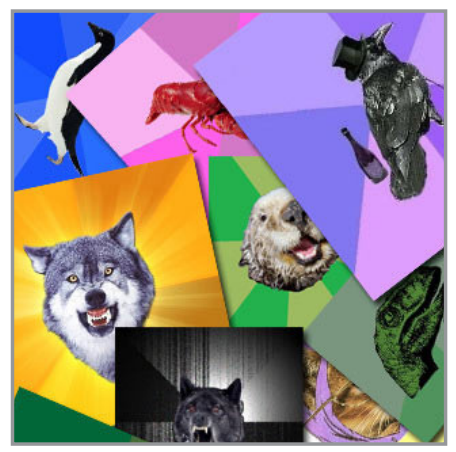

Illustration 7: Illustration de l'article "Advice Animals " . Source : KYM.

C'est la raison pour laquelle les personnages sont détourés, et que seul leur corps, voire leur tête reste sur l'image définitive. Les mèmes animaliers ont plus souvent recours à ces roues de couleur, comme le montre 
l'Illustration 7, et sont présentes dans un tiers des Advice Animals attestés sur KYM. La roue de couleur est une bonne façon de reconnaître le mème au premier coup d'œil, y compris sur un petit écran, et le texte écrit en lettres blanches liserées de noir est extrêmement lisible. Cette décontextualisation ne s'effectue pas uniquement sur les mèmes animaliers, comme en attestent "Wrong Lyrics Christina» et «Liberal Douche Garofalo» (Illustration 3).

D'autres méthodes peuvent servir la décontextualisation. Le mème «Minor Mistake Marvin» est né d'une photographie (Illustration 8), qui montre un petit garçon. Son visage à l'expression contrite est l'élément principal des dérivés, malgré le fait que sa contenance soit motivée par le micro-ondes dans lequel on peut présumer qu'il a fait brûler un pot de nouilles chinoises. Le mème prend son sens par cet accident, ainsi que son titre («Minor Mistake») bien qu'il soit exclu de l'image patron du mème, et qu'on ne fait que deviner le fond devant lequel l'enfant se trouve. Seul compte la mine du garçon, qui devient le porte-parole de toutes les situations qui provoquent la même expression. L'empathie entre le sujet de l'image et les coénonciateurs est un critère important, surtout pour les clichés représentés par des humains.

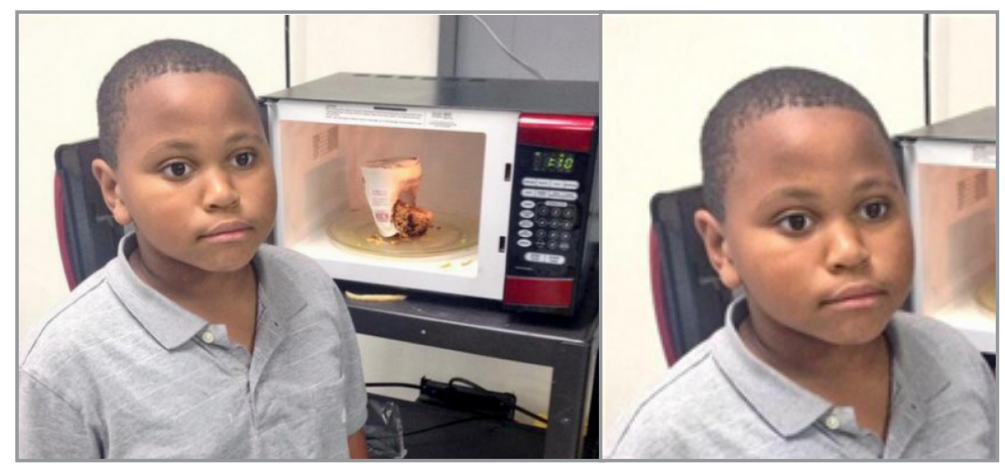

Illustration 8 : Image originale, et patron du mème «Minor Mistake Marvin» . Source : Imgur.

Ceci montre que les internautes ont en mémoire la situation originale dans laquelle la photographie a été prise, et que la décontextualisation par élimination sémiotique s'impose presque pour pouvoir utiliser ces illustrations comme patrons iconographiques. Créer un déficit dans l'image par l'exclusion d'éléments contextuels crée un terreau favorable à l'adjonction d'un discours stéréotypique, sujet de la deuxième partie de cette étude. La reconfiguration de la réalité iconographique est fréquente, 
et l'ensemble du dispositif image-légende-titre converge pour renforcer l'archétype mémétique.

\section{Discours}

Le genre employé differe selon le type de mème. Conseils ou parodies de conseils, microrécits autobiographiques, critiques à l'encontre de personnages du quotidien constituent les catégories principales des discours tenus. La première section de cette partie montre l'évolution des énoncés injonctifs, qui passe d'une recommandation à un instrument humoristique détaché de sa valeur normalement non fictionnelle. Les stéréotypes des Advice Animals servent également à parler de l'autre, qui n'est pas le coénonciateur, mais l'environnement commun du créateur et du lecteur. La critique de ceux qui les entourent (parents, professeurs et amis) sera l'objet de la seconde sous-partie. Enfin, je brosserai un portrait du discours personnel, dans des mèmes utilisés pour parler de soi.

\section{1. Émergence d'un nouveau discours injonctif}

Ces mèmes remettent en question les codes du genre prescriptif, ou injonctif-instructionnel (ADAM, 2011). La volonté d'amuser, voire de troller le lecteur en lui présentant des messages choquants est courante sur internet (MANDIBERG, 2012, p. 111), et certains personnages, nés sur 4Chan, s'illustrent par leur discours transgressif. Cette étude va dans la même direction que la constatation suivante, bien que la prescription atteigne ici une de ses limites : «En observant les lieux d'émergence du discours de consigne [...], on constate que celui-ci est transversal à différents domaines d'activité, mais aussi à différents types textuels et à différents genres discursifs" (MOURLHON-DALLIES, 2008). Les conseils visent normalement à faciliter une tâche, ou à améliorer des pratiques personnelles grâce aux recommandations de pairs. Les consignes données par ces Advice Animals ne ressemblent que formellement à ceux de ces discours. Les mèmes internet constituent un lieu d'humour et de connivence culturelle, où la visée illocutoire injonctive n'est qu'illusion, court-circuitée par l'omniprésence de l'absurde.

Les mèmes d' "Advice Dog" ", l'Advice Animal original, représentent à la fois de l'évolution de l'injonction vers la fiction humoristique, et des

${ }^{9} 787000$ résultats sur Google au 20 juin 2016. 
thèmes abordés dans ce genre mémétique. En effet, les recommandations de cet archétype sont absurdes et vont à l'encontre des valeurs morales (incitation aux meurtres, à la prise de drogues...).

La section «illustrations» de la page de ce personnage sur KYM contient 249 images. La répartition des thèmes évoqués dans ces représentations est exposée dans la Figure 1 :

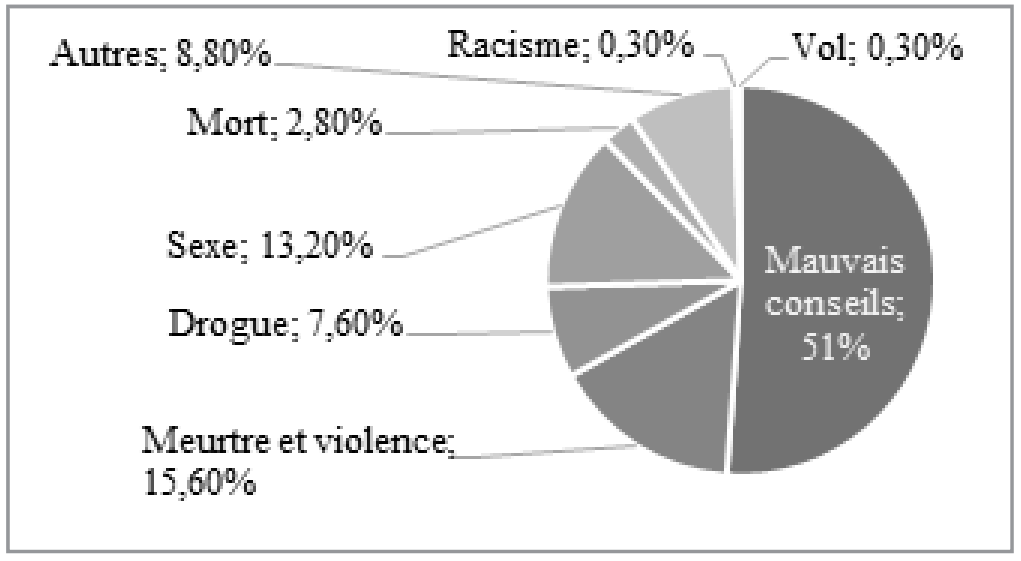

Figure 1 : Contenus évoqués dans les mèmes "Advice Dog» de la page KYM afférente au 20 juin 2016.

La catégorie la plus représentée est celle du mauvais conseil, c'est-à-dire celui qui contrevient à la morale, tout en ne recommandant pas une action répréhensible, à l'inverse des autres groupes. Appartient à cette catégorie le premier exemple de l'Illustration 9, «Scientology / The financial boost you need !» ('La Scientologie/ le coup de pouce financier qu'il te faut!'). 


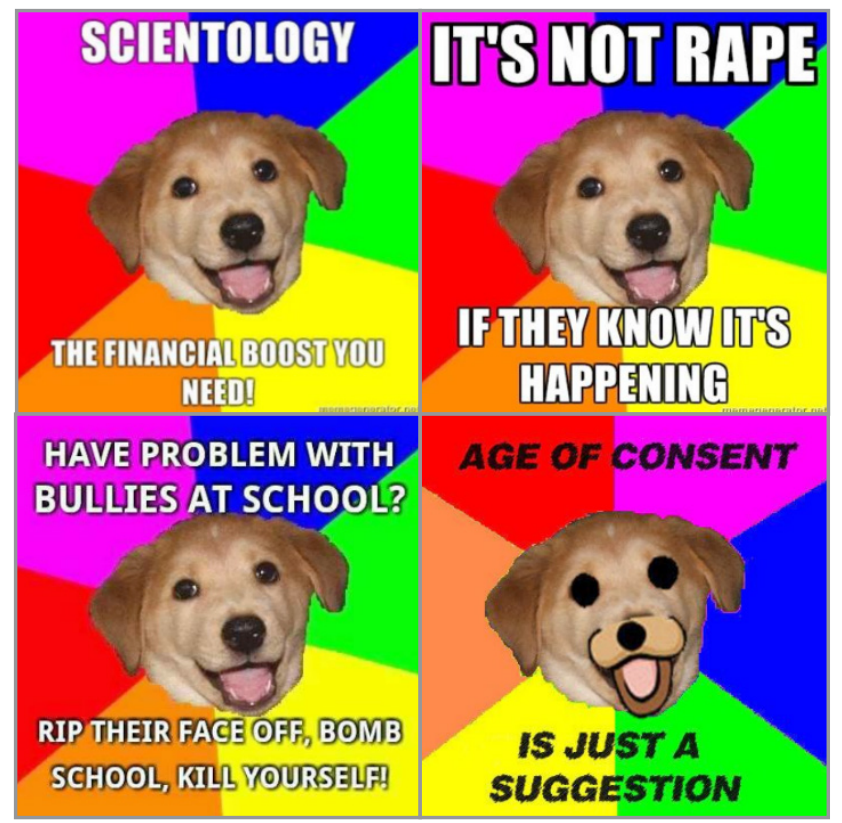

Illustration 9 : Exemples de mèmes d' «Advice Dog» .

Source : KYM.

Drogues, violence (Illustration 9b) et harcèlement à l'école (Illustration 9c) sont autant de préoccupations importantes dans la société états-unienne contemporaine. C'est certainement la raison pour laquelle ces thèmes sont abordés et même recommandés ironiquement dans ces mèmes. L'humour de l'Illustration 9-c tient au fait que la première partie «Have problems with bullies at school ?" ('Des soucis d'harcèlement scolaire?'), qui pourrait effectivement arriver à des lecteurs de mèmes, présente une résolution injonctive disproportionnée, illégale et violente, "rip their face off, bomb school, kill yourself" ('défigure-les, fais exploser l'école, suicide-toi). Léloignement axiologique de ce conseil avec les préconisations attendues crée le décalage absurde qui fait partie du cahier des charges d'un mème d'"Advice Dog".

La force illocutoire injonctive est parfois sous-jacente: «Age of consent/ is just a suggestion" ('la majorité sexuelle/ n'est qu'un conseil', Illustration 9d). Un argument à l'encontre de l'existence d'une telle majorité justifie l'acte prohibé (avoir des rapports avant la majorité sexuelle). Ces énoncés sont des incitations fictionnelles, appuyées par l'utilisation des yeux et de la bouche 
de Pedobear ${ }^{10}$, qui hybride l'image et donc le message iconique d'"Advice Dog» en [mauvais conseil] + [thème pédophile].

Si on reprend cette liste de critères pragmatiques de la recommandation : «visée illocutoire injonctive, place énonciative vide destinée à être occupée par le lecteur, monde représenté non-fictionnel» (ADAM, 2011, p. 98), la transgression ne touche que le dernier paradigme. L'impossibilité physique et la dangerosité placent ces conseils hors du réel, dans des cas comme «Suck helium/Defy gravity" ('Prends de l'hélium/Brave la gravité). Ils contribuent à la création d'un texte humoristique, sans lien avec une vraie préconisation, et comprennent un élément offensant, dans les thèmes abordés qui appartiennent au tabou (recours à la violence, incitation à la consommation de drogue...). La prescription n'est pas le seul type de discours de ces mèmes. Les genres particulièrement représentés sont la critique parodique de l'autre et la confession personnelle.

\subsection{Parodies critiques}

Les archétypes représentés peuvent être des critiques sociétales à lire au premier degré, de personnages du quotidien stéréotypés. C'est la raison pour laquelle nombre d'entre eux dressent le portrait de l'entourage des créateurs de ces mèmes (Figure 2). Aucun d'entre eux n'utilise un autre mode que la parodie de comportements jugés comme agaçants, et tournés en dérision par les auteurs de ces mèmes.

Le thème «autres" regroupe les acteurs du quotidien comme le propriétaire de son appartement, "Landlord Bear», ou l'adolescent d'un environnement protégé qui fait semblant d'être un dur ( Suburban Hardass", 'caïd de la banlieue'). Peu de ces mèmes parodient les femmes, mais ils comportent des archétypes comme "Irrational Black Woman", figure de la femme noire aux attentes contradictoires, dominatrice envers son compagnon. Les stéréotypes masculins abordés concernent surtout la masculinité hégémonique, à l'instar d' "Overly Manly Man" ('homme ultraviril).

\footnotetext{
${ }^{10}$ Personnage fictionnel d'ours pédophile né sur 4Chan, sujet de nombreuses blagues et mème à part entière.
} 


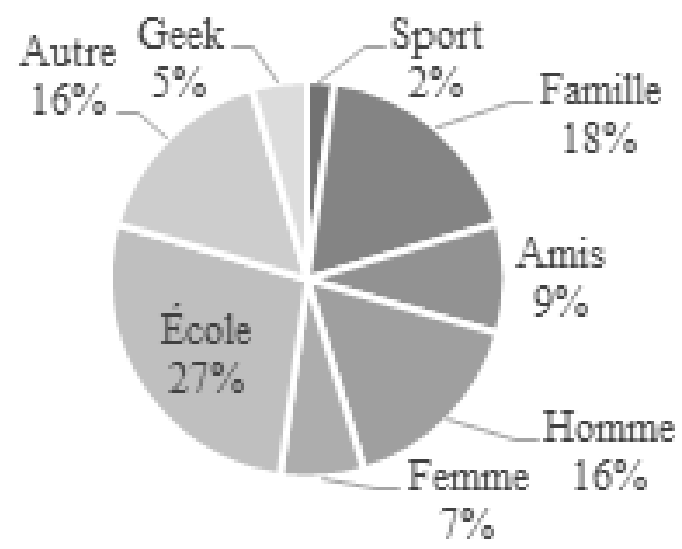

Figure 2 : Répartition des thèmes de mèmes parodiques.

Les thèmes qui touchent à la quotidienneté, le monde de l'éducation et les proches, totalisent chacun $27 \%$ des entrées de KYM (quand on additionne les catégories «famille» et "amis»). Les exemples étudiés ici seront «Engineering Professor», "High Expectation Asian Father» et «Annoying Childhood Friend» (Illustration 10). Ces mèmes exploitent respectivement le cliché du professeur peu utile et aux attentes disproportionnées ; celui du père asiatique jamais content des résultats de ses enfants ; et du copain d'école gâté et agaçant. 


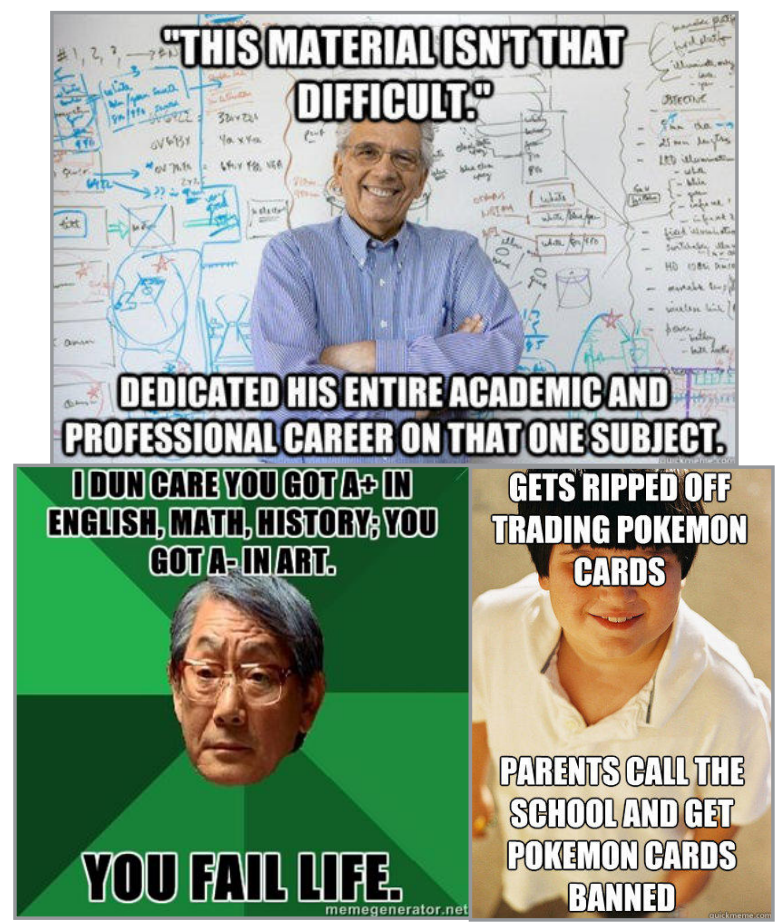

Illustration 10 : Mèmes des catégories «école», «famille» et «amis». Source : KYM.

Les commentaires des internautes sur leurs pages confirment qu'ils connaissent des exemples hors-ligne de ces personnes : «As an engineering student, I can conform [sic] this is true (dammit teachers!).» ('En tant qu'étudiant ingénieur, je peux confirmer que tout est vrai (bravo, les profs!)' "Engineering Professor»); "Im [sic] an Asian, and yes my parents just do like this to me. and i [sic] approve this meme. Back to studying" ('Je suis Asiatique, et effectivement, mes parents se comportent ainsi avec moi. Et je confirme ce mème. Au boulot? " High Expectation Asian Father "); "my childhood was crappy because of this" ("Mon enfance était pourrie à cause de ça" "Annoying Childhood Friend»). Ces mèmes servent d'exutoire aux concepteurs comme aux lecteurs. Ils prennent le pouvoir sur ces acteurs de leur quotidien en utilisant l'humour, et confirment leur existence en tant que mèmes grâce à leur authenticité hors-ligne. Le discours qui leur est attribué perd ici sa valeur initiale, énervante pour les coénonciateurs, pour devenir parodique une fois inséré dans le dispositif iconotextuel. 


\subsection{Récits personnels}

Les mèmes d'Advice Animal peuvent aussi servir d'espaces quasidiaristes, où les créateurs se cachent sous l'anonymat offert par une représentation fictive (et parfois animalière) pour se livrer. "Confession Bear " permet un exutoire de réappropriation mémétique puisqu'il remet en cause la culture hégémonique (VICKERY, 2014), notamment à cause de discours d'ordinaire tabous (homophobes, racistes, sur l'abus de drogue...).

Je parlerai donc ici de quelques cas de récits personnels sur la plateforme Reddit, et dans la sous-section r/Advice Animals. Les trois mèmes (Illustration 11) sont tirés de sa première page ${ }^{11}$, et sont tous des microrécits d'événements ponctuels, introduits par un message (au-dessus du mème). L'image sert à catégoriser le type de contenu de chaque mème, et agit donc comme une sorte de «hashtag contextuel», reconnaissable au premier coup d'œil, qui donne le ton du récit. «Seal of Approval»" aussi appelé «Satisfied Seal» ('Phoque euphorique') illustre le premier, et le second a recours à "Success Kid" ('Enfant triomphant'). Seul le premier mème serait compréhensible sans l'introduction du message, qui précise les circonstances de l'action. Les récits mémétiques débordent du cadre iconotextuel, et ce dernier sert de point d'ancrage thématique.

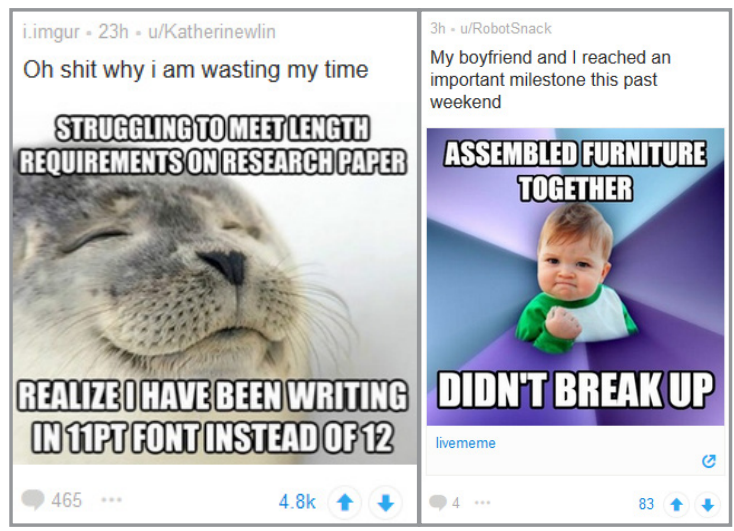

Illustration 11 : Exemples de mèmes postés dans la section «Advice Animals» . Source : Reddit (27 juin 2016).

\footnotetext{
${ }^{11}$ Ces mèmes sont disponibles à l'adresse : <https://m.reddit.com/r/AdviceAnimals>. Dernier accès le : 29 juin 2016.

12 Jeu de mots entre l'homographie de «seal», qui signifie "sceau» ou "phoque» : "seal of approval» signifie « approbation».
} 
La variabilité du cliché influence l'appui iconographique de ces microrécits. "Seal of Approval» et "Success Kid» sont des concurrents thématiques, qui représentent respectivement [satisfaction] et [triomphe ${ }^{13}$ ]. Les sentiments, ou une approximation de ceux-ci sont exprimés dans la seconde partie du discours. Pour "Seal of Approval», la scriptrice s'est rendu compte qu'elle avait écrit un devoir dans une police trop petite alors qu'elle avait du mal à produire un article assez long, et s'en apercevoir l'a soulagée; pour "Success Kid», l'utilisateur a pu monter des meubles avec son compagnon sans qu'ils rompent, ce qui provoque ce sentiment de triomphe. L'explication textuelle est indispensable, et l'émotion finale du scripteur passe entièrement dans l'image. C'est la raison pour laquelle le dispositif iconotextuel forme une entité infrangible, d'autant plus que la page de garde de la sous-section permet de voir les contributions sous la forme [message + miniature de l'image].

S'ils ont toujours la même forme scindée entre le haut et le bas de l'illustration, les discours tenus par les Advice Animals sont très différents. Qu'ils soient conseils mal intentionnés, exutoires aux frustrations provoquées par figures d'autorité et autres personnages du quotidien, ils sont le plus souvent parodiques et humoristiques. Ce n'est pas le cas des mèmes de récits anecdotiques, où les internautes utilisent les images comme méthode de classification de leur discours, à la manière des mots-dièse thématiques (ZAPPAVIGNA, 2015). Bien que ces images ne facilitent pas la recherche, contrairement aux mots-dièse, ils en simplifient le repérage grâce à leur archétype reconnaissable.

\section{Archétypies}

Dans cette partie, il sera question de la façon dont les archétypes mémétiques en ligne se distinguent et font ainsi évoluer ceux qui existent hors-ligne. Les stéréotypes animaliers évoluent en se renouvelant, preuve et conséquence de leur popularité. La subversion aux stéréotypes dans les mèmes qui représentent des humains est le sujet du second volet de cette partie.

${ }^{13}$ La mine fanfaronne de l'enfant exprime également la frustration, selon KYM. 


\section{1 Évolution de stéréotypes}

La tradition culturelle n'impose pas ses stéréotypes aux Advice Animals. Les animaux en tant que tels n'incarnent pas une vertu ou un défaut, à la manière de leur utilisation dans certains genres fictionnels. On s'éloigne donc dans ces mèmes des récits où l'animal doué de parole symbolisait une valeur (par exemple, la ruse du goupil dans Le Roman de Renart, ou les personnages des fables ésopiques).

Le même animal peut servir à plusieurs représentations, comme "Courage Wolf», et son dérivé "Insanity Wolf» (Illustration 12), tous deux issus de 4Chan en 2006 et 2009 respectivement. Les photographies de loups utilisées changent peu : attitudes menaçantes, têtes détourées et adjonction de roue de couleur, la décontextualisation y advient de la même façon.

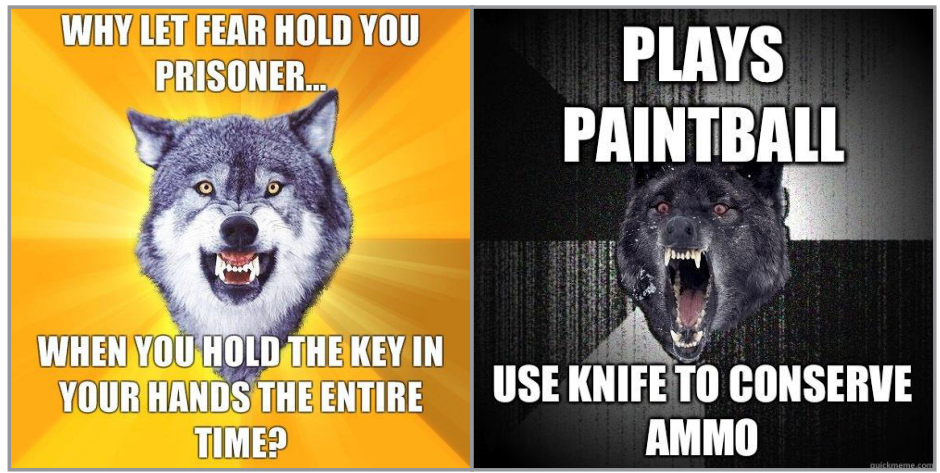

Illustration 12 : Exemples des mèmes "Courage Wolf» et "Insanity Wolf» . Source : KYM.

Pourtant, le choix d'une teinte chaude pour "Courage Wolf», et d'un dégradé de gris pour "Insanity Wolf» distingue dès le premier coup d'oeil le type de discours qui y est tenu. "Insanity Wolf» comporte des éléments sémiotiques qui aident à sa différenciation : allure plus agressive, fourrure sombre, une roue de couleur grainée le borde. Il n'est pas auréolé par le fond, contrairement à "Courage Wolf». Bien que l'animal ne change pas, le respect de sèmes iconographiques traditionnels garantit l'éloignement thématique des deux archétypes. Les énoncés qu'ils portent les séparent : motivation personnelle centrée autour de la notion de courage, contre discours violent. Le loup est une figure plurivoque dans deux dispositifs très proches. 
Il est fréquent dans la culture mémétique d'utiliser un seul et même animal pour exprimer des idées opposées : "Socially Awkward Penguin", créé en 2009, s'est décliné un an plus tard en «Socially Awesome Penguin» (Illustration 13). Iconographiquement, les deux éléments divergents sont la direction de l'illustration de pingouin, et la teinte de la roue. Ils représentent les archétypes opposés [gaucherie] et [charisme].

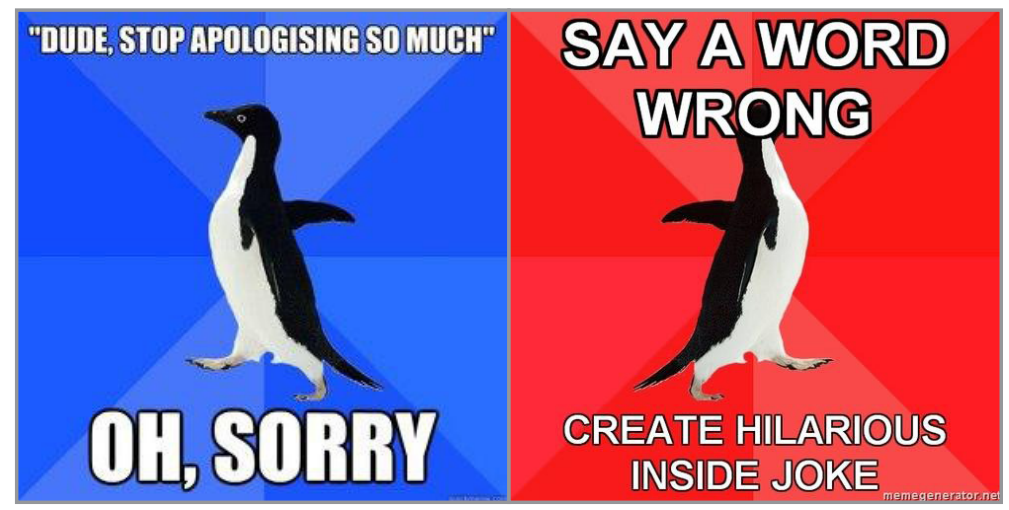

Illustration 13 : Exemples des mèmes «Socially Awkward Penguin " et "Socially Awesome Penguin».

Source : KYM.

Un animal choisi incarne donc un des éléments du paradigme, et il peut contenir en son sein son propre opposé, toujours dans un but humoristique. Les personnages dérivés sont compréhensibles même sans connaissance de l'Advice Animal prime, mais leur lien de parenté s'impose pour les internautes qui partagent de cette culture. Le renversement conceptuel de ces mèmes secondaires est la seule solution employée dans ce corpus pour maintenir une relation sémantique forte avec l'original.

La distanciation avec l'idée reçue originelle est aussi au cœur des Advice Animals qui mettent en scène des stéréotypes humains.

\subsection{Subversion aux stéréotypes humains}

Dans la section 2.2, les mèmes à l'étude représentaient des personnages du quotidien tournés en dérision, à cause de l'archétype qu'ils représentaient. Ce sont des figures de repoussoirs, desquelles l'imitation 
seule suffit à provoquer le rire. Elles se distinguent des subversions aux idées reçues par rapport à certaines minorités états-uniennes, si l'on prend «Normal Guy» (Illustration 14a : 'regarde le sport à la télélne fait pas de sport') comme archétype de personne la plus ordinaire possible, représentant donc la majorité, voire les dominants ${ }^{14}$.

Le cas de «Successful Black Man» sera examiné ici. La dichotomie discursive façonne la dynamique entre l'amorce, raciste, car faisant appel à des stéréotypes, et la chute, qui neutralise ce message.
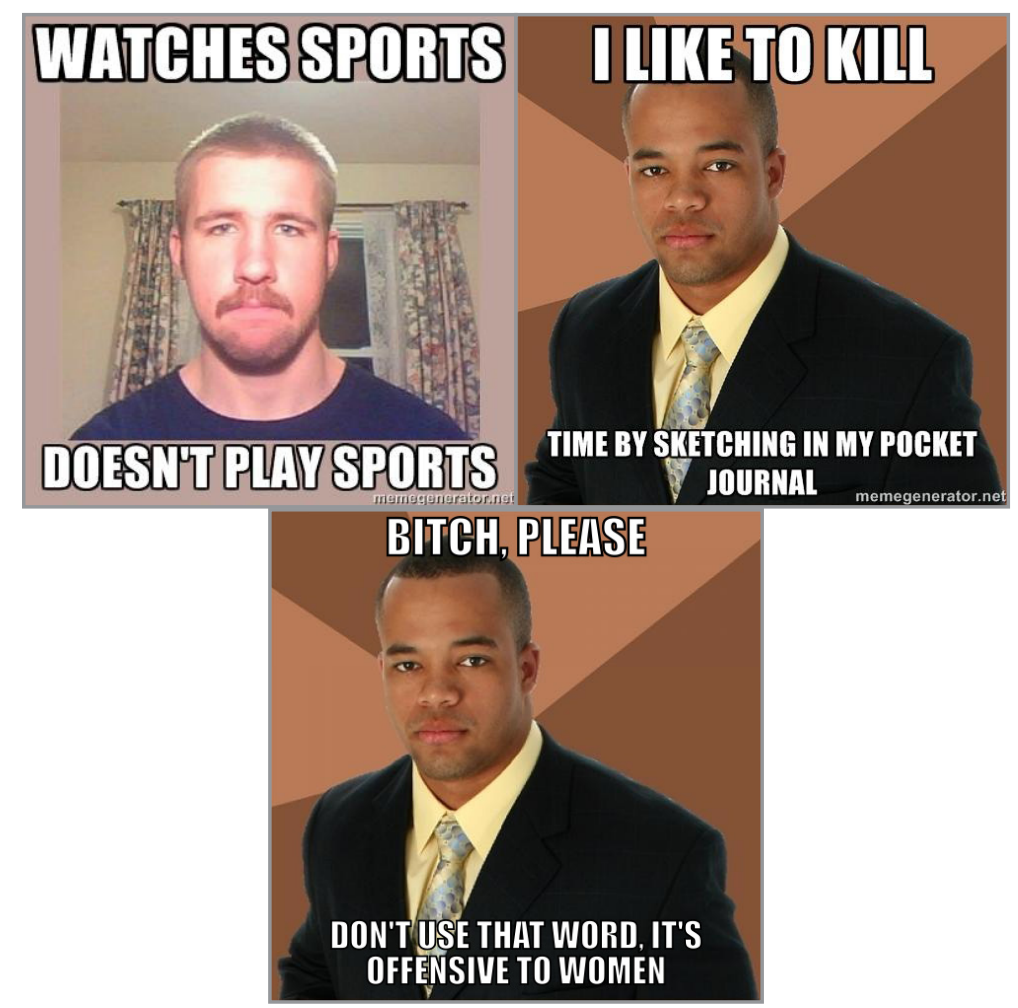

Illustration 14 : Exemples de «Normal Guy» et de «Successful Black Man». Source : KYM et Memegenerator.

L'organisation spatiale des mèmes provoque cette distance entre l'amorce et la chute. Écologiquement, ce type de discours s'appuie sur le scrolling, c'est-à-dire le fait que l'illustration apparaisse à l'écran de haut

${ }^{14}$ Ce personnage se caractérise par ses actions tout à fait banales, sans aucun intérêt.

fragmentum. Santa Maria: Programa de Pós-Graduação em Letras, UFSM, n. 48, 
en bas, quand on fait défiler les images. Le principe du "bait and switch" ('appât et substitut), plus souvent associé au trolling, intervient ici, puisque le début et la fin du discours se contredisent et qu'ils trompent les horizons d'attente des lecteurs.

Les thèmes abordés dans l'amorce constituent un inventaire de stéréotypes racistes envers les Afro-Américains : performances physiques et sexuelles, drogues, allusions à l'esclavage, violence, nourriture ${ }^{15} \ldots$ Les mèmes de "Successful Black Man» sélectionnés ici (Illustration 14b et c) montrent des exemples de conduite agressive, physiquement et verbalement ('J'aime tuer / le temps en dessinant dans mon carnet'; 'Putain ${ }^{16}$, vas-y / n'utilise pas ce mot. Il est misogyne'). Le dispositif imagier est aussi ambivalent, et sert le discours. La teinte du fond rappelle la couleur de peau de l'homme alors que son costume évoque la prospérité, et son expression faciale neutre ne permet pas de trancher pour l'un ou l'autre des deux qualificatifs qui lui sont associés. Grâce à la syntaxe de l'anglais qui place les adjectifs objectifs plus près du nom-tête que les adjectifs subjectifs ${ }^{17}$, la tension mémétique se lit aussi dans la construction du titre. Le [[Successful] [Black man]] ${ }^{18}$ est d'abord un homme noir (amorce) sur lequel on applique une série de stéréotypies, puis un homme qui a réussi (chute contradictoire).

Le racisme et la xénophobie sont exprimés plus directement en ligne, grâce à l'anonymat relatif qui permet simultanément de s'ouvrir et de ne pas se dévoiler (VICKERY, 2014, p. 323). C'est l'écart entre un «imaginaire» moralement répréhensible, car raciste, et l'allotopie du discours de la seconde partie qui crée le décalage humoristique, ingrédient déterminant pour la perpétuation du mème. Ce type de mème prouve que ce racisme existe toujours, puisque ces plaisanteries font appel à des stéréotypes encore inscrits dans le substrat culturel, bien qu'ils y soient remis en cause.

\section{Conclusion}

Le dispositif des mèmes permet de donner le plus d'informations dans le plus petit espace possible. L'image est sémiotiquement dense pour figurer un archétype identifiable d'emblée, que le titre renforce encore, surtout

\footnotetext{
${ }^{15}$ Le poulet (le plus souvent frit) et la pastèque sont les deux aliments les plus souvent associés au stéréotype raciste sur les Afro-Américains.

${ }^{16}$ Littéralement, "chienne, s'il te plaît», "bitch please» est un idiome très courant en anglais vernaculaire.

${ }^{17} \mathrm{La}$ règle générale est adjectif subjectif puis ceux de taille, âge, forme, couleur, origine, matière.

${ }^{18}$ Schéma mèmonymique identique à celui de [Ordinary [Muslim man]] évoqué en 1.2.
} 
pour les mèmes où figurent des photographies d'humains. Ces mèmes offrent un espace discursif dans lequel déverser un discours stéréotypique pourtant sans cesse renouvelé, d'où la notion de "cliché variable», qui impose la réactualisation et la publication de mèmes, tout en maintenant le cadre thématique initial. L'archétype repose sur le lien indissociable entre l'être figurant sur l'image, le fond, et les types d'énoncés qui y sont tenus. Elle est garantie et conservée par le titre.

Les clichés variables vont à l'encontre d'une série d'horizons d'attente discursifs. Les Advice Animals remettent en question la dialectique injonctive, bienveillante et normalement censée promouvoir une attitude positive, les recours aux animaux pour incarner une valeur morale, et les préconceptions racistes. Les internautes y positionnent leurs microrécits personnels grâce à l'image immédiatement reconnaissable. Plus que des personnages d'archétypes, à la substance invariante, la perspective thématique dicte l'emploi de ces figures. Utiliser un Advice Animal pour parler de soi constitue une sorte d'antonomase iconographique.

Lidentité même du stéréotype présenté peut évoluer grâce au lien conceptuel d'opposition entre le dérivé et l'original, pour renouveler un genre ou un personnage apprécié. Les mèmes remettent en question les préconstructions sociales, en utilisant le biais de l'humour pour les courtcircuiter, grâce au dispositif, ou au retournement discursif effectué dans la chute.

\section{Referências}

AGAMBEM, Giorgio. Qu'est-ce qu'un dispositive? Rivages Poche. Petit Bibliothè, 2014.

BARTLETT, Jamie. The Dark Net. Random House, 2014.

CLARK, Sean. An Hero. The Troll Cave. Publié le 20 février 2011. Disponible à l'adresse: <http://punk1119-thetrollcave.blogspot.fr/2011/02/ hero.html>. Dernier accès le : 14 juin 2016.

DAVIES, Mark. The Corpus of Contemporary American English: 520 million words, 1990-present. 2008. Disponible à l'adresse : <http:// corpus.byu.edu/coca/>. Dernier accès le : 29 juin 2016.

GEACH, Peter. Ontological relativity and relative identity. In: MUNITZ, Milton K., Logic and Ontology. New York, New York University Press, 1973. 
HUFFMAN, S.; OHANIAN, A. Reddit. 2005-. Disponible à l'adresse : <www.reddit.com>. Dernier accès le : 30 juin 2016.

JOUXTEL, Pascal. Les mèmes dans le dictionnaire Larousse, Société francophone de mémétique. Publié le 25 septembre 2015. Disponible à l'adresse : <http://www.memetique.org/2013/09/les-memes-dans-lelarousse-2014/\#more-1334>. Dernier accès le : 10 juin 2016.

KIM, Brad (Ed.). Know your Meme. 2008-. Disponible à l'adresse : <http://knowyourmeme.com/>. Dernier accès le : 30 juin 2016.

MANDIBERG, Michael. The Social Media Reader. NYU Press, 2012.

PAVEAU, Marie-Anne. Les prédiscours. Sens, mémoire, cognition. Paris : Presses Sorbonne nouvelle, 2006.

POOLE, Christopher. 4Chan. 2003-. Disponible à l'adresse : <www.4chan. org $>$. Dernier accès le : 30 juin 2016.

RASTIC, A.; DAZDAREVIC, S.; FIJULJANIN, F. New language media: internet memes manuscripts. UNITE : v. 1, n. 2, p. 38-44. 2014.

SCHAAF, Alan. Imgur, 2009-. Disponible à l'adresse : <www.imgur.com>. Dernier accès le : 27 juin 2016.

SHIFMAN, Limor. Memes in Digital Culture. MIT Press, 2014.

VICKERY, Jacqueline. R. The curious case of Confession Bear: the reappropriation of online macro-image memes. Information, Communication \& Society, v. 17, n. 3, p. 301325. 2014.

ZAPPAVIGNA, Michele. Searchable talk: The linguistic functions of hashtags. Social Semiotics, v. 25, n. 3, p. 274-291. 2015. 